\title{
E-ART: LA FRAMMENTAZIONE E IL MONTAGGIO
}

\begin{abstract}
Alessandro Zinna*
ABSTRACT: Considerare la scrittura elettronica come un linguaggio? Roman Jakobson rispondeva positivamente solo dopo aver constatato la presenza di due funzioni: quella metalinguistica e quella estetica. La scrittura elettronica è sottoposta qui al vaglio delle due condizioni del linguista russo: per essere tale il linguaggio elettronico deve poter parlar di se stesso e creare degli effetti estetici che gli sono propri.

Appurato che si tratta di un linguaggio, il saggio ha coma obiettivo di capire quali innovazioni introduce l'arte nata dalla scrittura digitale. Facendo ricorso alle intuizioni di Deleuze e Guattari che opponevano l'arte alla filosofia e alla scienza - si possono cogliere le proprietà costitutive di quelle che Foucault chiama, disponendole sullo sfondo delle evoluzioni storiche, le formazioni discorsive. Apparsa nel primo volume di Electronic Literature, Self Portrait(s) [as other(s)] è un'opera esemplare per riassumere le innovazioni introdotte. Prendendo spunto dal dispositivo ideato da Talan Memmott, i tratti metalinguistici ed estetici sono letti sullo sfondo delle evoluzioni della formazione dell'arte. Dalle proprietà del piano, della pratica e del valore sono ricostruite le mutazioni e le tendenze dell'arte elettronica. II saggio si conclude aggiungendo una terza condizione che precisa quelle enunciate da Jakobson: l'intraducibilità degli effetti estetici nei linguaggi delle arte.
\end{abstract}

PAROLE CHIAVE: Arte digitale. Montaggio. Testo potenziale. Formazioni semiotiche. Autore.

\section{Premessa}

Bisogna riconoscere che la scrittura elettronica non ha avuto la giusta risonanza nello studio delle scienze del linguaggio. Verso questa scrittura gli studiosi hanno assunto due atteggiamenti. II primo consiste nel ridurla a un modo di manipolare l'espressione considerandola, di fatto, alla stregua di un altro strumento di scrittura o di un medium appena più sofisticato. II secondo atteggiamento sembra invece orientato ad accordarle una qualche dignità, per quanto marginale, che è poi quella riconosciuta ai linguaggi.

*Médiations-Sémiotiques-LERASS, Université de Toulousa II - Jean Jaurès, Toulouse, France. alessandro. E-mail: zinna@univ-tlse2.fr

Esta obra está licenciada com uma licença Creative Commons. 
Lo scarso interesse e, di conseguenza, il deficit di ricerca che per molti anni ne è seguito, sono stati piuttosto sorprendenti soprattutto se consideriamo che questa scrittura introduce l'interattività come un modo convenzionale di produrre i documenti digitali, dando origine a testi e dispositivi interattivi. Qual è di fatto l'importanza di includere la scrittura elettronica tra i linguaggi e, soprattutto quando è legittimo farlo? Jakobson diceva che siamo di fronte a un linguaggio quando si verificano due condizioni: un linguaggio deve poter parlare di se stesso, ma soprattutto, per essere tale, un linguaggio deve poter generare un'estetica che non esisterebbe senza quel linguaggio. Comincerò con l'assumere questa argomentazione per mostrare come la scrittura elettronica, proprio quella che esplora la sua specificità estetica, possieda tutti i connotati richiesti per essere un linguaggio.

\section{Le formazioni dell'Arte, della Filosofia e della Scienza}

Deleuze e Guattari, avevano cercato di identificare i tratti inerenti al genere filosofico arrivando ad opporlo a due altri generi: da una parte il discorso scientifico, dall'altra al discorso artistico ${ }^{2}$. Per caratterizzare quelle che, ricorrendo a Foucault, possiamo identificare come le due formazioni discorsive in grado di delimitare gli estremi del discorso filosofico, gli autori si appoggiavano sul riconoscimento di alcune proprietà, a partire da quelle che appartengono al piano ${ }^{3}$.

La scienza ci parla di qualcosa che c'è già, di fatto non inventa niente. II suo discorso può descrivere, classificare, perfino predire, ma non può creare i suoi propri oggetti. Per esercitare la sua pratica ha bisogno di un piano di referenza. La scienza si riferisce sempre a qualcosa che le preesiste ed è questo qualcosa a costituire in definitiva il suo piano di referenza. D'altra parte la filosofia quell'attitudine propria degli studi che nella Grecia antica prelude all'atteggiamento scientifico - nasce dal distacco dal piano della trascendenza. Per spiegare un

\footnotetext{
2 Rimandiamo a Deleuze e Guattari (1991).

${ }^{3}$ Rimandiamo a Foucault (1969) e alla rilettura che ne abbiamo riproposto (Zinna 2012).
} 
evento la filosofia crea un piano d'Immanenza evitando il ricorso a fattori magici o religiosi e ricercando le sue ragioni nella causalità immanente. L'arte infine, esattamente all'opposto della scienza, inventa ciò che non esiste ancora. Dandole un'esistenza, fa che l'opera sia: il suo piano è quello della creazione ${ }^{4}$. Un'altra proprietà, distinta dal piano, è per i due filosofi quella della pratica. Il discorso della scienza ha come pratica la ricerca delle funzioni, opportunamente distinte in costanti e variabili; la filosofia invece stabilisce una varietà di concetti; l'arte, infine, per il suo modo di operare porta a far variare i percetti. Infine, l'ultimo tratto distintivo è il valore che viene perseguito. Riducendo all'essenziale gli argomenti dei due filosofi possiamo riassumere dicendo che la scienza ha come finalità la verità, la filosofia l'interessante, e l'arte il bello.

Evidentemente la contrapposizione proposta de Deleuze e Guattari è una semplificazione estrema di problematiche ben più complesse. Ma proprio per questa identificazione della formazione discorsiva attraverso il piano, la pratica e la finalità, diventa più facile cogliere le trasformazioni a cui stiamo assistendo. Un limite consiste nel fatto che, fissando le condizioni essenziali per ciascuna formazione, gli autori non tengono conto del loro sviluppo storico. Seguendo invece Foucault e la sua idea di archeologia delle formazioni possiamo constatare che una prima trasformazione riguarda il valore su cui giudichiamo l'arte. Osservando le mutazioni del genere possiamo accorgerci che rispetto al valore attribuitole per esempio da Lessing nel Laocoonte, l'arte contemporanea non insegue più da tempo il bello dei percetti inteso come armonia delle forme, ma ha assorbito piuttosto il valore che contraddistinguere la filosofia (LESSING, 1766). È facile infatti accorgersi che nel dominio dell'arte è soprattutto l'interessante a essere perseguito attraverso la proposizione di concetti. Nell'arte concettuale quella che comincia con Duchamp - diventa un tratto caratteristico che si affermerà sempre di più nel tempo mostrando che il valore perseguito della formazione artistica si è vistosamente spostato verso l'interessante concettuale. Molta dell'arte contemporanea d'altronde sarebbe del tutto insignificante se la si

${ }^{4}$ Seguendo Foucault, in un precedente scritto, avevo proposto di chiamarle formazioni semiotiche. 
giudicasse con un metro diverso, per esempio in base a un'estetica del bello o, di converso, alla legittimità del brutto nell'arte, quali proposte già da Lessing e ribadite di recente da Eco $(2007 ; 2010)$. Già dal Novecento, a dominare le finalità dell'estetica non è più l'assiologia del bello e del brutto. Questa tendenza non fa che accentuarsi nel nuovo millennio. L'interessante è anzitutto concettuale e solo secondariamente è o può essere rilevante dal punto di vista percettivo. Dall'arte concettuale in avanti si è cominciato a produrre una riflessione senza necessariamente porsi come obiettivo la finalità estetica del bello o del suo contrario. Proprio come avviene in filosofia: l'arte ci incita a interrogarci su dei concetti. Di fatto essa non ci parla più della bellezza o non lo fa più come un valore vincolante, ma ci propone una riflessione sulle tematiche che le sono esterne o ci invita a riflettere sull'arte stessa in quanto genere o linguaggio. Liberandosi dal valore del bello, l'arte ha dunque accolto questo smottamento di valori e temi portandoci a pensare alle condizioni stesse della sua produzione.

Una delle tematiche preferite dal discorso dell'arte a partire dal Novecento è proprio quella di parlarci del modo in cui l'arte stessa ci parla. Rivisitando periodicamente le sue regole compositive, l'arte pone alla riflessione la domanda su cos'è e come si produce arte. II Novecento è dominato da questa tendenza che attraversa la pluralità delle sue formazioni semiotiche, non solo la pittura o la letteratura, ma ugualmente il cinema, il teatro e più di recente l'arte digitale. Tematizzando il modo stesso di produrre e dunque di dire arte, come avviene per ogni funzione metasemiotica, l'arte prende a oggetto se stessa e i suoi canoni. Ecco allora apparire la prima delle due funzioni poste da Jakobson per definire un linguaggio: la funzione metalinguistica diventata dominante. L'E-Art non soltanto non smentisce questa tendenza ma ne fa, come vedremo, un suo tratto costitutivo. Tutte le proprietà che possiamo elencare, il fatto stesso di aver superato la problematica della riproducibilità, così cara a Benjamin, per esempio l'aver azzerato la dialettica tra l'originale e la copia, quella d'aver privilegiato l'interessante sul bello, e infine di porre come tema dominante il linguaggio stesso - per esempio quello combinatorio, come a suo tempo ha fatto l'OULIPO costituiscono nient'altro che un completamento di quel percorso ideale delle 
tendenze artistiche dell'ultimo secolo. Resta da chiederci se l'arte elettronica introduca degli elementi di novità e se possieda un suo modo specifico di produrre il senso tali da farne un linguaggio a parte. Ricercando il secondo tratto jakobsoniano possiamo adesso domandarci se non ci sia dunque una maniera propria ai linguaggi elettronici di produrre gli effetti estetici o di lavorare altrimenti alcuni dei tratti riconosciuti alla formazione dell'arte come per esempio quello della variazione seriale.

\section{Self Portrait(s) [as other(s)]}

Per rispondere a quest'ultimo interrogativo proporrò alcune osservazioni sull'opera ipertestuale di Talan Memmott, apparsa nella raccolta Electronique Litterature Collection (volume one) col titolo Self Portrait(s) [as other(s)]

Guardando la pagina d'accesso possiamo cominciare col chiederci quanti quadri vediamo in questo primo quadro, ovvero quanti dispositivi geometrici distinguiamo sullo sfondo nero che li accoglie (Fig. 1)?

Figura 1. La pagina d'ingresso

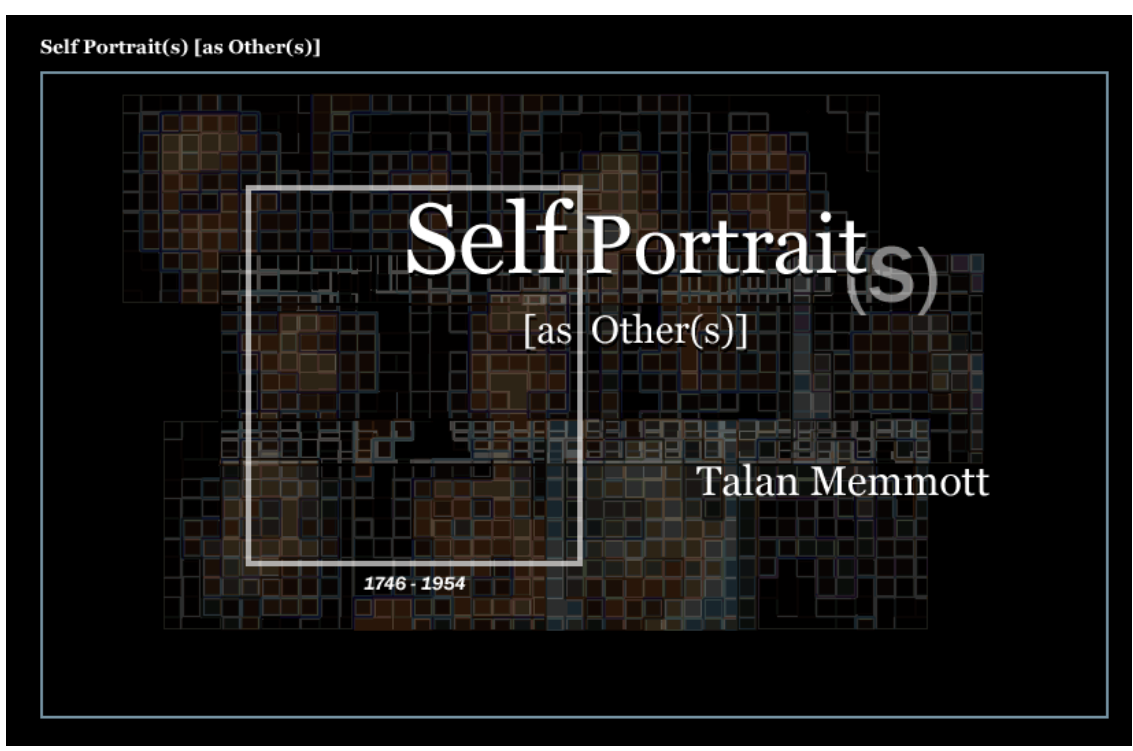

7 Avendo già presentato un'analisi particolareggiata del dispositivo dell'opera di Memmott in occasione del Congresso dell'Associazione Francese di Semiotica di Lione (cfr. Zinna 2010), mi limiterò qui a riassumerne alcuni tratti salienti. 
Anzitutto un bordo bianco che delimita un primo quadro rispetto a un fuori quadro dove compare il titolo Self Portrait(s) [as other(s)]. Al suo interno una divisione in piccoli quadri, a forma di pixels, che ricompongono le immagini di autoritratti celebri. Infine un quadro bianco in primo piano che seleziona una zona in cui appaiono, sullo sfondo, delle parti di differenti ritratti.

Se passiamo alla schermata successiva (Fig. 2), se entriamo all'interno del dispositivo, ci troviamo di fronte a un quadro composto da due moduli: un modulo per l'immagine, che si trova a sinistra, e uno sulla destra predisposto ad accogliere la scrittura. II quadro che contiene le immagini, si presenta a prima vista come il risultato di un'operazione di montaggio: dei frammenti di autoritratti, piuttosto noti, e, al tempo stesso, una serie di interventi grafici e di manipolazioni elettroniche di pixellizzazione. II modulo a destra presenta invece una descrizione la cui disposizione tipografica ricorda la nota biografica. Così, se l'immagine a sinistra è supposta raffigurare l'autoritratto del pittore, la scritta che appare a destra ne costituisce la nota biografica. Da qui l'impressione di essere in presenza di una scheda informativa accompagnata dal ritratto o, piuttosto, come indicato dal titolo stesso dell'opera, dall'autoritratto dell'artista. Già a un primo sguardo, il dispositivo di Memmott mantiene la promessa annunciata nel titolo: quella di enunciare se stesso come altro(i). 
Figure 2. Un esempio di composizione

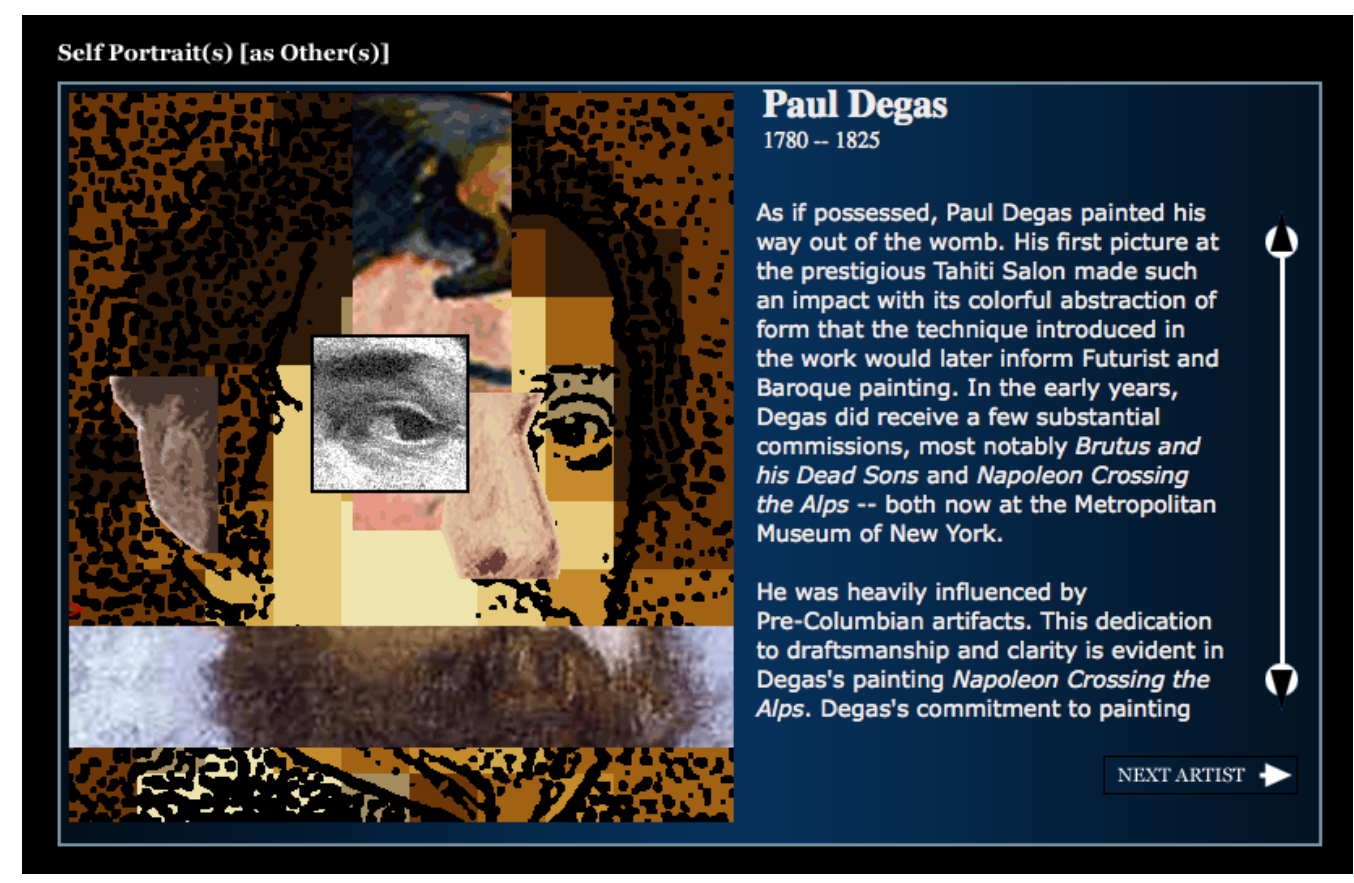

\section{II dispositivo}

Diamo brevemente uno sguardo alla struttura interattiva dell'opera. A prima vista un principio di frammentazione è introdotto nella composizione stessa delle schede.

All'interno di ogni schermata si possono distinguere degli ordini di gerarchia di montaggio. A un primo livello troviamo due diversi montaggi per frammenti: uno non-lineare, quello dei ritratti, e uno lineare, quello delle parti verbali. Ma possiamo ancora distinguere un secondo tipo di montaggio che chiameremo multimodale, dato dalla connessione tra le informazioni visive e quelle verbali; per finire, un ultimo tipo di montaggio, gerarchicamente superiore, è quello delle schede stesse. Presa visione di quella che si presenta infatti come la prima scheda, il lettore non può che proseguire nella sola direzione concessa, cioè avanzando da una scheda all'altra grazie al pulsante NEXT ARTIST. La successione che ci viene offerta ci indica la possibilità di percorrere queste schede secondo un ordine apparente di successione. Un indizio interessante però è costituto dal fatto che l'autore non ha previsto alcun pulsante per tornare alla scheda precedente. Così se attiviamo 
ripetutamente il pulsante NEXT ARTIST ci accorgeremo che non c'è modo di tornare all'immagine di partenza. Questo perché il movimento che dovrebbe far avanzare le schede secondo una successione lineare ha una funzione ben diversa. II pulsante ha il compito di mischiare in modo aleatorio i frammenti già predisposti in memoria: da una parte variando i frammenti dei quadri che si alternano dentro ai rettangoli in cui è stata scomposta l'immagine; dall'altra i frammenti di cui si compone la nota biografica scritta sia a livello del nome che del contenuto. II presunto avanzamento, in realtà, determina una duplice mutazione nel contenuto dei due moduli. È d'altronde facile constatare la non coincidenza tra il nome e il cognome dell'artista dall'invenzione di autori immaginari come "JeanAuguste Degas" o "Pierre-Auguste Goya". La regola implicita sembra quasi quella di evitare la coerenza tra le due parti che formano il nome proprio di un autore. Anche qui, come nell'immagine, riconosciamo una parte inserita in un tutto che le è estraneo. La frammentazione non tocca solo il nome, poiché concerne al tempo stesso la composizione dell'immagine visiva $e$ il nome, cioè i due elementi che determinano rispettivamente l'identità figurativa e quella nominale del pittore. Questa sorta di Arcimboldo composto per autoritratti, sia da frammenti autobiografici che da nomi-cognomi di pittori conosciuti, è generato dal motore in cui sono stati scomposti rispettivamente i ritratti e i nomi dei pittori. Per questa ragione, dopo aver visionato molte schede, ci accorgiamo non soltanto che non c'è ripetizione, ma che non riusciamo a scorrere l'intera successione perché non esiste alcuna successione e neppure un percorso lineare. Sebbene, presentata come un'interattività sintagmatica, il pulsante NEXT ARTIST determina delle commutazioni paradigmatiche tra i frammenti predisposti in memoria.

\section{La struttura generativa}

Ciascuna delle operazioni di commutazione a cui il lettore da origine con il suo gesto è possibile perché una stessa posizione all'interno dei dispositivi non-lineari e lineari può essere occupata da una classe di elementi: nel nostro caso, da una parte dalla classe di frammenti di autoritratti e dall'altra dai frammenti verbali. Se vogliamo scoprire la struttura algoritmica delle commutazioni, bisognerà cercare 
nell'immagine e nelle scritture le posizioni in cui commutano i frammenti. Ora, se possiamo constatare che il nome si compone di due sole posizioni in cui si trovano a variare dodici nomi di altrettanti pittori, risulta più difficile determinare lo schema delle posizioni in cui commutano i frammenti visivi.

Ci accorgiamo allora che il viso è scomposto in diversi moduli e che questi moduli sono, per ciascun ritratto, da quattro a cinque. Globalmente le posizioni dividono il viso in categorie di frammenti per altrettanti dettagli: l'occhio sinistro, quello destro, il naso, l'orecchio sinistro o destro, il mento e la bocca. Tra questi moduli è soltanto quello orizzontale in basso a rimanere costante poiché non cambia né di posizione né di taglia, e a breve ne cercheremo la ragione. La forma degli altri moduli invece non conserva sempre la stessa posizione e può subire delle modificazioni e delle variazioni nella forma (Figg. 3-4). È facile constatare che il naso e l'orecchio per esempio appaiono spesso scontornati e talvolta alcuni di questi moduli sono sovrapposti per ottenere una maggiore coerenza figurativa. Infine, ci accorgiamo che la totalizzazione dei frammenti visivi avviene sulla base di un dispositivo di tratti grafici a disegno con la funzione di unificare i diversi frammenti dando loro un contorno unitario (Fig. 5).

Figura 3. I moduli dove commutano i frammenti visivi

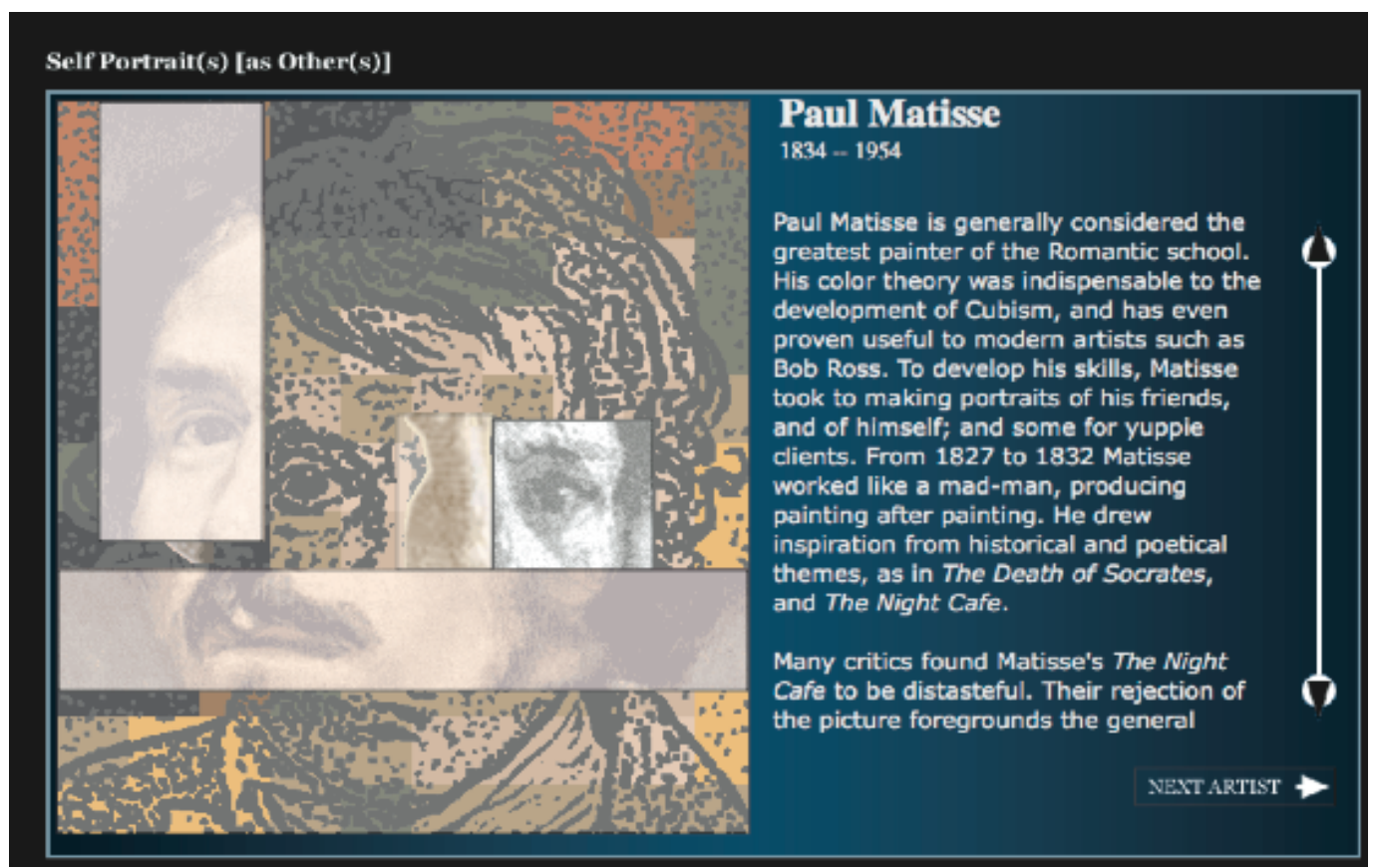

Texto Digital, Florianópolis, Santa Catarina, Brasil, v. 11, n. 2, p. 35-52, jul./dez. 2015. ISSNe: 1807-9288. 
Figura 4.Ancora una variante dove commutano i frammenti visivi

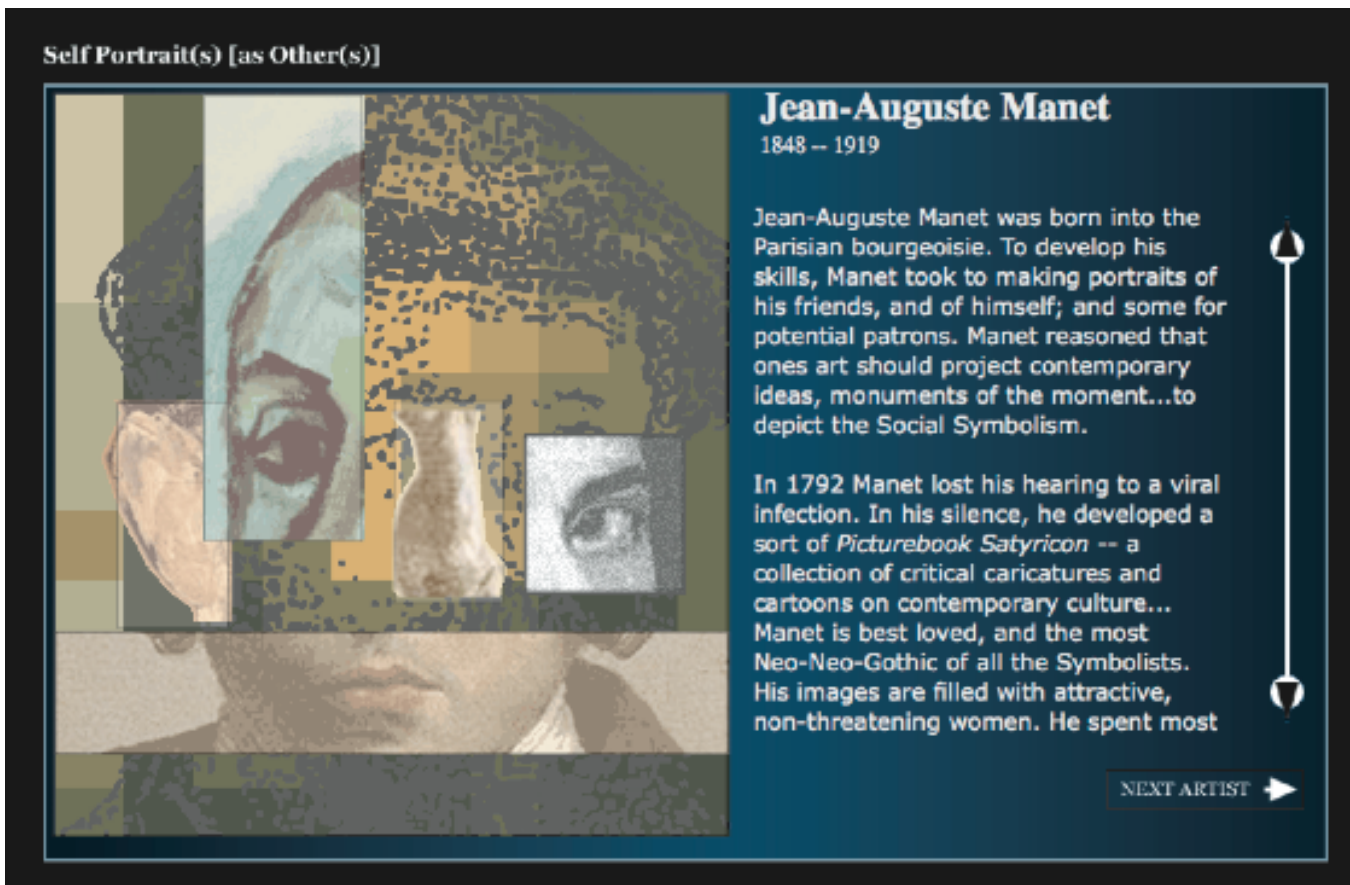

Figura 5. II modulo orizzontale costante all'altezza della bocca

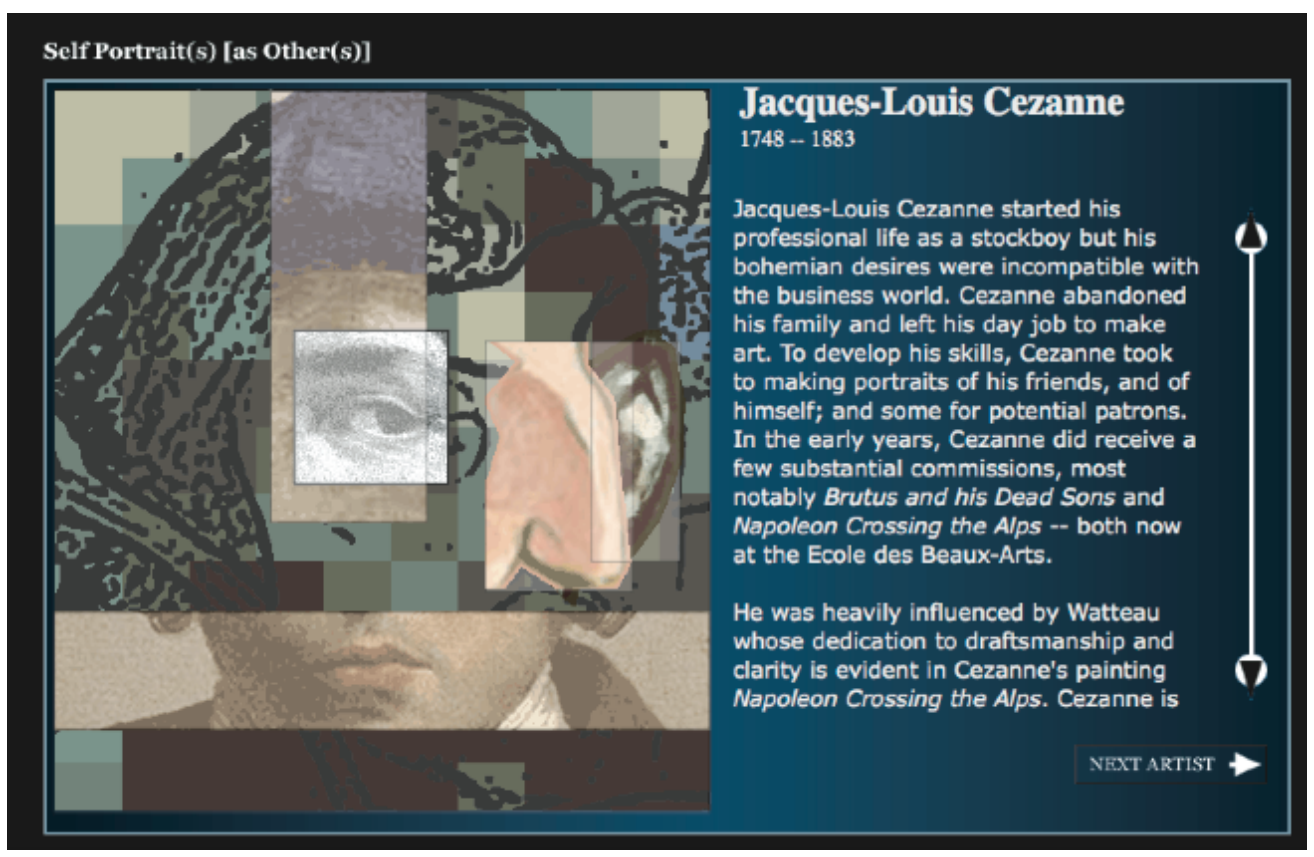

Texto Digital, Florianópolis, Santa Catarina, Brasil, v. 11, n. 2, p. 35-52, jul./dez. 2015. ISSNe: 1807-9288. 
Figura 6. Altro esempio di modulo orizzontale che inquadra la bocca

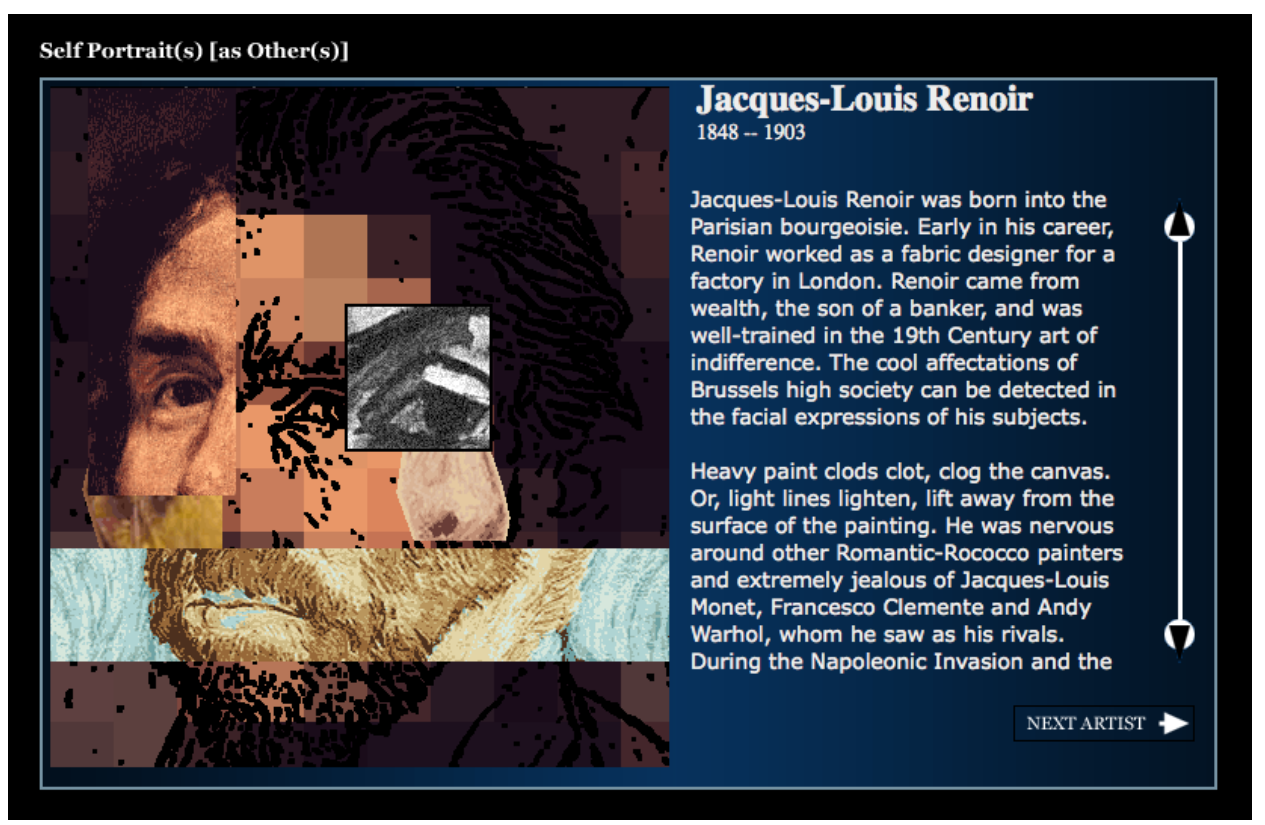

\section{Dare la parola}

II pulsante NEXT ARTIST non è la sola interattività concessa al lettore. Se infatti spostiamo il cursore sul modulo orizzontale, cioè sull'unico modulo che non cambia di posizione né di taglia, l'immagine ci svela una sorta di messaggio nascosto (Figg. 8-9).

Figura 7. La rivelazione di un pensiero come una frase detta

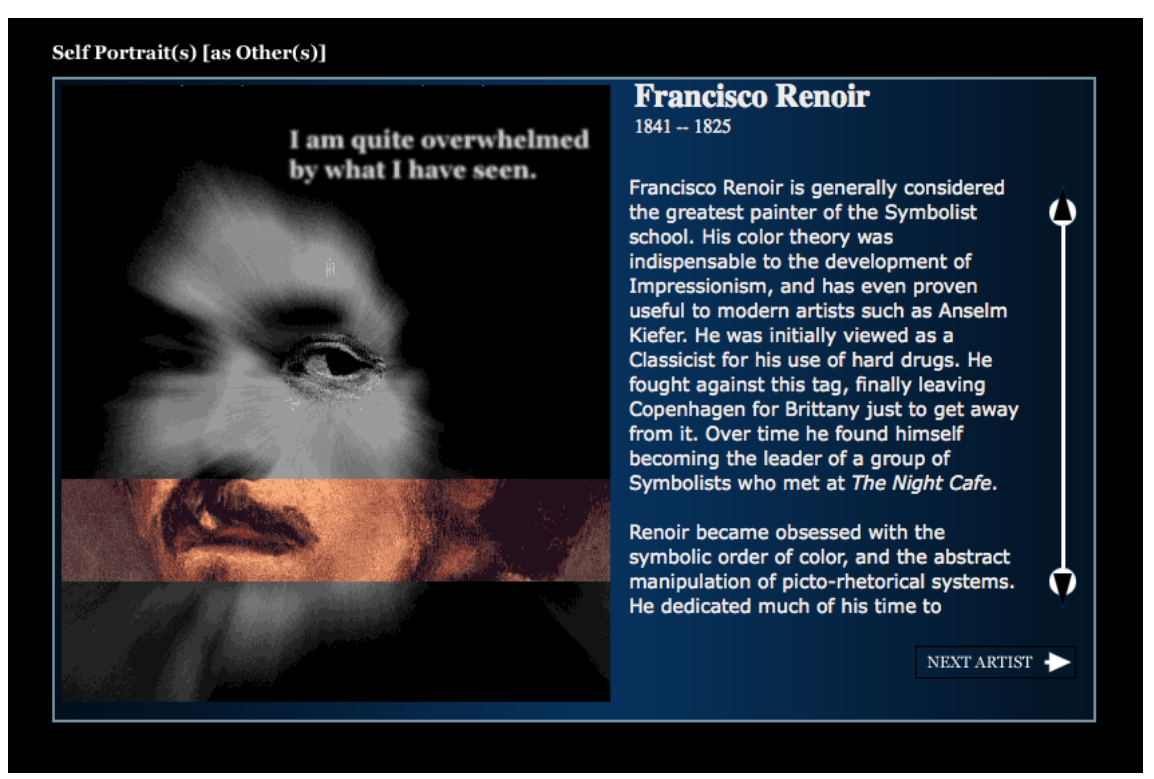

Texto Digital, Florianópolis, Santa Catarina, Brasil, v. 11, n. 2, p. 35-52, jul./dez. 2015. ISSNe: 1807-9288. 
Figura 8. Un'altra rivelazione di un pensiero come una frase

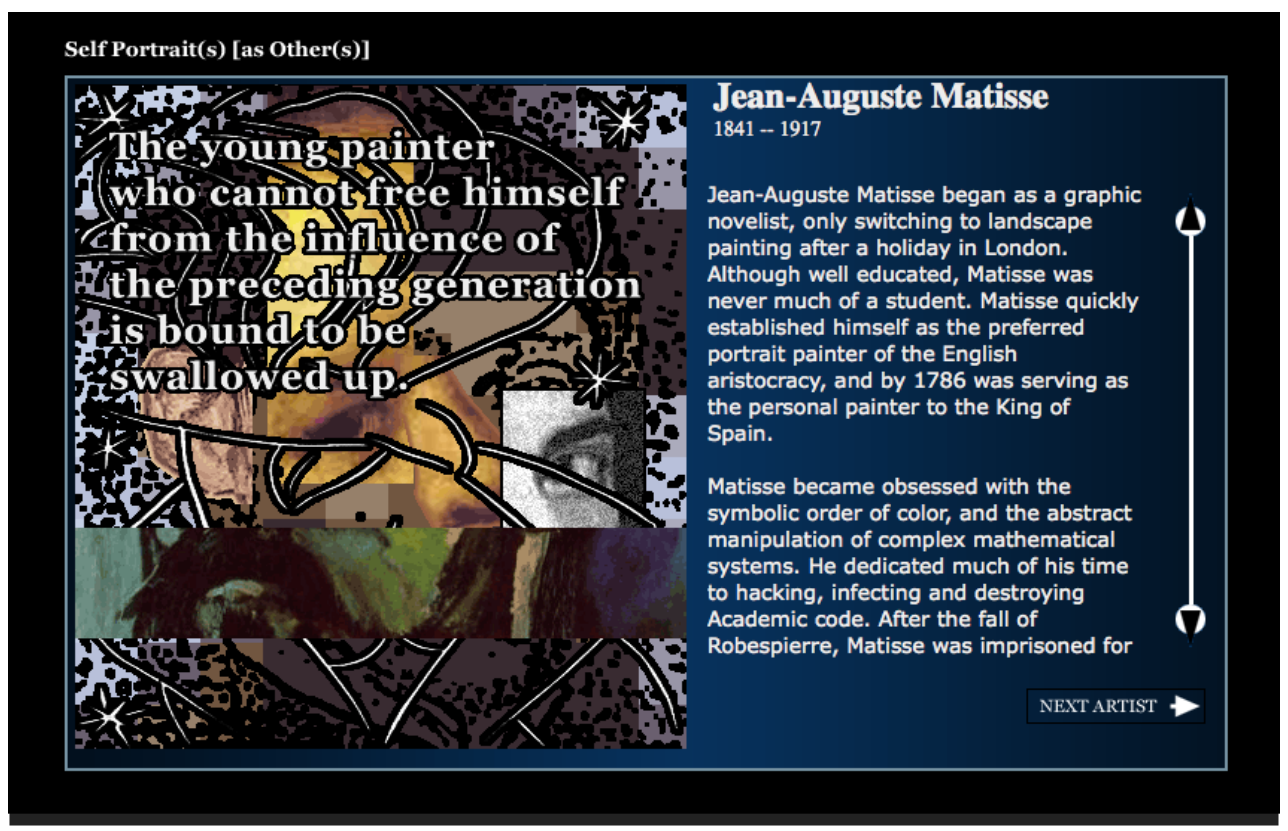

L'attivazione del segmento produce un cambiamento che riguarda l'autoritratto: introducendo un nuovo elemento verbo-visivo, la composizione ci mostra una breve frase che sembra esprimere un frammento di pensiero come una verità personale dell'artista. La zona da cui si attiva questa interazione è quella in cui si alternano i frammenti delle bocche. Non soltanto l'azione del lettore combina i frammenti dell'autoritratto attraverso il gesto interattivo del pulsante NEXT ARTIST, ma attraverso questa seconda interazione, il lettore sembra dare la parola al ritratto svelando un pensiero nascosto del pittore.

\section{L'autore e il suo dispositivo}

II dispositivo che abbiamo descritto, in maniera certo succinta, ci invita a interrogarci anzitutto sul ruolo dell'autore e del lettore nell'arte. È stato spesso osservato che la scrittura elettronica attribuisce un ruolo cruciale al lettore ipertestuale.

Nel dispositivo di Memmott, in maniera paradossale, l'identità visiva e verbale che dovrebbe essere composta dall'autore stesso, è determinata almeno due volte da 
altro(i). Sul piano dell'enunciato lo è dai frammenti composti da altri pittori, su quello dell'enunciazione attraverso i gesti del lettore che determinano la loro combinazione. Sul piano dell'enunciato l'opera di Memmott è a) una riflessione non banale sull'identità del pittore nella pratica dell'autoritratto in pittura; al tempo stesso è b) una critica dell'identità attribuita per il nome proprio e la biografia dell'autore; e infine, c) una critica al genere prodotto dalle schede biografiche come discorso proprio della Storia dell'Arte. Ma soprattutto, sul piano dell'enunciazione, l'opera diventa una riflessione sull'identità dell'autore di un testo interattivo. In definitiva possiamo concludere che è proprio la natura dell'enunciazione nell'arte elettronica che si trova ad essere tematizzata nell'opera di Memmott.

Une simile tematizzazione sembra voler fissare essenzialmente due concetti: da una parte che l'autoritratto in pittura è il risultato di una scrittura collettiva: un pittore è sempre la risultante dei pittori che lo hanno preceduto; dall'altra che l'identità del pittore è determinata dal lettore, poiché di fatto è il lettore, grazie al suo gesto interattivo, che gli attribuisce un viso, un nome, nonché una storia e perfino un pensiero. In questa vertigine paradossale di dirsi in un autoritratto, ma attribuendo al lettore il compito di stabilirne di volta volta l'identità, possiamo chiederci qual è il ruolo dell'istanza autoriale che si trova ad assumere la sua totalità l'enunciazione del dispositivo ipertestuale. In altri termini, chiedersi dov'è l'autore e in che senso si tratta di un Selfportrait as other(s)? Se questa domanda ha un senso è perché non può essere l'autore della realizzazione di ogni singolo testo a assumere un simile ruolo, almeno in quanto questo ruolo è già occupato dal lettore. Quello che identifichiamo come l'autore dell'ipertesto diventa piuttosto l'istanza che ha prodotto l'intero dispositivo non più come un testo fissato e realizzato nella sua invarianza, ma piuttosto come un testo potenziale capace di generare di volta in volta tutte le singole occorrenze. Queste occorrenze sono, al tempo stesso, degli atti di produzione (generazione di una disposizione di frammenti) e quindi di interpretazione del risultato (dato da ogni singolo percorso dei frammenti attualizzati). Con la differenza che, se comunemente il lettore di un ipertesto sceglie tra i dei percorsi disponibili, come tra una serie di alternative 
predisposte dall'autore, qui la scelta coincide con la mossa aleatoria del lettore stesso che si trova a creare il percorso grazie a un coup de dès che è affidato alla macchina enunciativa. Ora, mentre nello scenario più tradizionale la scelta tra queste alternative spetta al lettore, nell'iperteso di Memmott è il gesto di lettura delle schede che determina la produzione di ogni singola scheda.

Una prima conseguenza che possiamo trarre è che molte opere e dispositivi ipertestuali introducono una distinzione tra testo realizzato e testo potenziale. L'autore del testo realizzato è generalmente il lettore, mentre l'autore è l'istanza che produce il testo potenziale, come tale capace di assumere tutte le varianti che potranno essere prodotte dalle differenti combinazioni.

\section{Conclusioni}

Avevamo cominciato questa riflessione con due osservazioni di Jakobson sul linguaggio e una di Deleuze e Guattari sull'arte. Si è in presenza di un linguaggio, diceva Jakobson, quando sono rispettate due condizioni: la capacità di parlarci di se stesso e la capacità di creare un'estetica specifica. Se la prima proprietà ci porta a riconoscere la possibilità che ha il linguaggio elettronico di parlare di se stesso, la seconda condizione nasce invece dalla capacità di creare dei testi potenziali in una stessa opera. D'altra parte ci accorgiamo dell'impossibilità di tradurre l'estetica del linguaggio elettronico in un altro linguaggio proprio perché alcuni di questi effetti estetici non potrebbe esistere fuori da quel linguaggio. Possiamo tradurre i linguaggi tra loro, ma non le estetiche che sono prodotte in

questi linguaggi. È facile accorgersi che non possiamo tradurre il dispositivo ipertestuale di Memmott in nessuna altra successione di segni linguistici, in nessun altro linguaggio diverso dal dispositivo ipertestuale ideato per visionarlo. L'estetica esplora i limiti di un linguaggio facendoci conoscere, di volta in volta, le specificità di un sistema. Questa stessa impossibilità di tradurre gli effetti estetici porta in realtà a riconoscere il livello di adeguazione del dispositivo al senso, mostrando senza equivoci che la scrittura elettronica non è la trasposizione del piano dell'espressione da un sistema di segni a un altro, ma un nuovo linguaggio 
espressivo in senso pieno. L'adeguazione estetica tra il contenuto e il sistema d'espressione elettronica resisterebbe ai tentativi di trasporre questi effetti in un altro sistema di segni. Proprio perché non è una mera proprietà del contenuto, gli effetti estetici prodotti nel linguaggio elettronico appaiono intraducibili.

D'altra parte abbiamo visto che, almeno secondo Deleuze e Guattari, l'arte opera una continua variazione dei percetti. Questa idea di messa in variazione come esigenza stessa dell'arte la troviamo non più nella pluralità delle realizzazioni artistiche, per esempio seriali, ma all'interno di una sola opera: è proprio questa continua variazione che fa della creazione elettronica un testo potenziale cioè un testo che contiene la molteplicità seriale delle sue messe in variazione. Una variazione ottenuta secondo un principio di autogeneratività che può essere, secondo i casi, forte o debole. Spinto all'estremo, il principio di autogeneratività porta alla generazione automatica del testo: l'enunciazione di ogni singola realizzazione è affidata alla macchina. Superando così l'ultima idea di autore come produttore di un'occorrenza o di una variazione e, in definitiva, persino quella del lettore come produttore del percorso, si introduce e si esalta quella che Couchot chiamava l'enunciazione macchinica, quell'on impersonale che possiamo attribuire alla macchina (COUCHOT, 2003).

C'è infine un'altra caratteristica peculiare al lavoro di Memmott. I frammenti che diventeranno il sistema di elementi compositivi sono la costituzione preventiva di uno stock, creato dalla frammentazione di un numero delimitato di elementi preesistenti, che determineranno tutte le possibili variazioni. Questa evidente decostruzione preventiva ci dice che la generazione del senso non avviene più attraverso la creazione di elementi che vanno dal più piccolo al più grande, ma per scomposizione di blocchi di senso e per il loro successivo montaggio. II senso preesiste già nei frammenti che saranno montati e non è più il risultato dell'aggregazione di atomi di senso. Ma quella parte dell'immagine che non vediamo rimane in qualche modo presente. Potremo dire che è latente nelle zone che sono occupate dai frammenti di altri autoritratti. L'atto di creazione diventa nell'opera il risultato prima di una negazione data per decostruzione, e in seguito 
un montaggio casuale delle parti. Se la decostruzione spetta all'autore dell'ipertesto, il montaggio aleatorio richiede il gesto del lettore che stabilisce quella che possiamo considerare una variazione seriale stocastica.

Questa idea di riassumere l'arte e i meccanismi della creazione artistica in una sola opera diventa un tratto prevalente nell'estetica dell'E-Art. L'autore si sposta dalla parte di dio, non crea più testi, ma macchine e dispositivi enunciativi che, col contributo interattivo del lettore, e quello non meno secondario della macchina, generano le occorrenze dei singoli testi, delle singole varianti 0 , più modestamente, della serie minimale di variazioni. Non si crea più un'opera, ma un dispositivo che produce tante opere quante sono le sue possibilità di messa in variazione grande o piccola che sia. Nel caso che abbiamo considerato, queste combinazioni, per quanto numerose, sono in numero finito. In altri casi, l'ambizione della variazione infinita prevale. C'è quasi un'attrazione fatale verso la generazione automatica e illimitata della variazione.

In fondo, come mostrano gli ultimi lavori di Eduardo Kac, una via possibile per questa riflessione sul linguaggio, capace di inventare la serie delle sue variazioni, è la creazione artistica su base biologica. È il tocco ultimo, quello che si appropria della mano che è attribuita generalmente a dio, capace di creare l'uomo che crea. In quanto rappresenta l'evoluzione dell'artista elettronico, Kac non tematizza più il dire meccanico, ma l'atto stesso di creare il senso organico. La creazione di variazioni percettive negli esemplari vegetali è ottenuta attraverso un'alterazione del codice biologico. A differenza della macchina elettronica, la struttura vivente non deve essere programmata perché segue già un suo programma: la variazione diventa piuttosto un'alterazione o un'interferenza nella programmazione della materia vivente (KAC, 2003). Proprio attraverso la creazione/variazione/alterazione genetica l'arte raggiunge la sperimentazione scientifica. Le barriere tra arte, filosofia e scienza diventano estremamente labili: queste formazioni cominciano a registrare delle mutazioni nell'ordine dei valori e soprattutto delle pratiche. II laboratorio non più è una metafora, ma la condizione che, sempre più spesso, la pratica dell'arte si trova a condividere con la scienza. 


\section{E-ART: FRAGMENTATION AND ASSEMBLY}

ABSTRACT: Electronic writing here is analysed through the two conditions set by the Russian linguist Roman Jakobson: in order to be that the electronic language must be able to speak of itself and to create its own aesthetic effects.

Inspired by the work of Talan Memmott Self Portrait(s) [as other(s)] metalinguistic and aesthetic traits are read on the background of the evolution of art. From the properties of the plan, the practice, and the value the changes and trends in electronic art are reconstructed. The article concludes by adding a third condition which specifies those set from Jakobson: the untranslatability of aesthetic effects in the languages of art.

KEYWORDS: Digital art. Assembling. Potential text. Semiotic formations. Author.

\section{Riferimenti bibliografici}

COUCHOT, Edmond. L'Art numérique. Comment la technologie vient au monde de l'art. Paris: Flammarion (in collaborazione con NORBERT Hillaire), 2003.

"De la communication à la commutation: l'art et le Web" Revue Ligeia Art et Multimédia —, n 45-48, luglio-dicembre, 2003.

DELEUZE, Gilles e GUATTARI, Felix. Qu'est-ce que la philosophie? Paris: Minuit (coll. « Critique »), 1991.

ECO, Umberto. Storia della bellezza. Milano: Bompiani, 2007. Storia della bruttezza. Milano: Bompiani, 2010.

FOUCAULT, Michel. L'archéologie du savoir. Paris: Gallimard, 1969.

JAKOBSON, Roman (1963). Saggi di linguistica generale, Milano: Feltrinelli, 1966. 
KAC, Edoardo. "Natural History of the Enigma", 2003-2009. Ultimo accesso 30 Ottobre, 2014. URL: <http://www.ekac.org/nat.hist.enig.html>.

LESSING, Gotthold Ephraim (1766). Laocoonte, ovvero sui confini tra poesia e pittura, Milano: Rizzoli, 1994.

MEMMOTT, Talan. "Self Portrait(s) [as other(s)]", 2003. Ultimo accesso 30 Ottobre, 2014. URL:

$<$ http://collection.eliterature.org/1/works/memmott_self_portraits_as_others/spo_ app/X00.html>.

ZINNA, Alessandro. "L'écriture hypertextuelle : Le cadre et les fragments", colloque International Les écritures fragmentaires, 2-4 décembre, Lyon, Association Française de Sémiotique, Actes du Colloque, 2010.

"Les formations sémiotiques", VS. Quaderni di studi semiotici, n 114 , Milano: Bompiani, 2012. pp. 127-147.

Recebido em: 19/10/2015. Aceito em: 03/12/2015. 